\title{
$\pi$-Conjugated Donor-Acceptor Systems as Metal-Free Sensitizers for Dye-Sensitized Solar Cell Applications
}

\author{
M. Wielopolski ${ }^{1}$, J.-E. Moser ${ }^{1}$, M. Marszalek ${ }^{1}$, S. M. Zakeeruddin ${ }^{1}$, and M. Grätzel ${ }^{1}$ \\ ${ }^{1}$ École Polytechnique Fédérale de Lausanne, Institute of Chemical Sciences \& Engineering, SB ISIC \\ GR-MO, Station 6, CH-1015 Lausanne, Switzerland
}

\begin{abstract}
High extinction coefficients and easily tunable spectral properties of $\pi$ conjugated donor-acceptor dyes are of superior advantage for the design of new metalfree organic sensitizers for applications in dye-sensitized solar cells. Ultrafast transient absorption spectroscopy on the femtosecond and nanosecond time scales provided deep insights into the dependence of charge carrier dynamics in fully organic dye/ $/ \mathrm{TiO}_{2}$ systems on i) the donor-acceptor distance, ii) the $\pi$-conjugation length, and iii) the coupling to $\mathrm{TiO}_{2}$ by different anchoring groups. Importantly, the observed differences in charge transfer dynamics justify the variations of photovoltaic performances of the dyes as applied in solar cell devices. This leads to the conclusion that the photoconversion efficiencies strongly depend on a delicate interplay between the dyes' building blocks, i.e. the donor, the $\pi$-conjugated spacer and the anchor/acceptor moieties, and may easily be tuned by molecular design.
\end{abstract}

Dye-sensitized solar cells (DSSCs) have attracted high interest due to their remarkable performances in converting solar energy to electricity at relatively low cost as compared with conventional siliconbased photovoltaic devices [1]. One of the key roles in DSSCs is attributed to the sensitizers, which are responsible for light absorption and the generation of electric charges. Among these, ruthenium sensitizers exhibit record solar to electricity power conversion efficiencies (PCEs) of $11-12 \%$ under AM $1.5 \mathrm{G}$ irradiation [2].

Currently, however, more and more research activities focus on the design and development of new metal-free organic sensitizers with the purpose to increase the PCEs $[3,4]$. The high extinction coefficients of organic dyes and the ease of tuning their spectral properties by standard synthetic methods turned out to be of superior advantage. Thereby, the most efficient photovoltaic performance was achieved when employing sensitizers of a donor- $\pi$-conjugated spaceracceptor/anchoring (D- $\pi-\mathrm{A})$ architecture. Lately, several synthetic strategies lead to the development of various donors based on coumarin, indoline, tetrahydroquinoline, triarylamine, heteroanthracene and carbazole with a cyanoacrylic acid as the acceptor/anchoring unit. DSSCs derived from this type of D- $\pi$-A systems have achieved up to $10 \%$ efficiency under AM $1.5 \mathrm{G}$ irradiation [3]. Even though many structural variations of the donor moieties have been reported the impact of the $\pi$-spacer and of the acceptor/anchoring groups has been much less explored - notwithstanding the fact that these parameters show a significant influence on the spectral response and device performance. Particularly with regard to increase the PCE values of fully organic DSSCs and outdistance the ruthenium based cells, the tunability of the electronic properties by the control of the conjugation length and the anchoring modes to $\mathrm{TiO}_{2}$ is of special interest [4].

This is an Open Access article distributed under the terms of the Creative Commons Attribution License 2.0, which permits unrestricted use, distribution, and reproduction in any medium, provided the original work is properly cited. 
Recently, we have contributed to this field by reporting on sensitizers using triarylamine donors and benzothiadiazole(BTDA)/cyanoacrylic acid acceptors (Fig. 1a). There, an additional phenyl ring induces an out-of-plane torsion of the adjacent BTDA and cyanoacrylic acid leading to an interruption of the $\pi$-conjugation between donor and anchoring group in the cationic state. This inhibits the back electron transfer while leaving the electron injection rate unaffected resulting in different lifetimes of the charge separated states and, hence, differences in the photovoltaic performance [5].

In another study we have investigated the phtoconversion efficiencies of a D- $\pi$-A-type dye containing cyanobenzoic acid as anchoring group. In this context we have emphasized the influence of the binding mode to $\mathrm{TiO}_{2}$ as imposed by the chemical structure of the anchor (Fig. 1b) [6].

a)

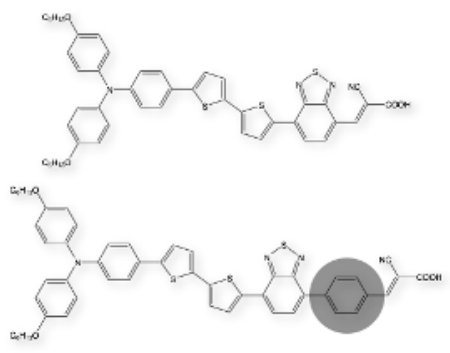

c)

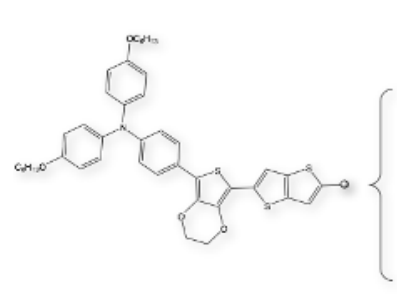

Fig. 1. a) Chemical structures of investigated sensitizers with triphenylamine donors and BTDA/cyanoacrylic acid acceptors. b) Side and front views of the binding modes of a cyanobenzoic acid to $\mathrm{TiO}_{2}$. c) Chemical structures of sensitizers with various benzoic acid derivatives as acceptor/anchoring groups.

To seize the impact of a variation of anchoring modes in D- $\pi$-A systems new organic sensitizers with various benzoic acid derivatives as acceptor/anchoring groups have been synthesized and tested in DSSCs. Thereby, we investigated the substitution of the cyano and/or acetylene bond on the phenyl group and the impact on the optical properties and photovoltaic device performance [6]. All structures of the new sensitizers (coded as KM-1, KM-2, KM-3, KM-5 and KM-6) are represented in Fig. 1c. The incorporation of benzoic acid was expected to extend the $\pi$-conjugation and promote a relatively planar structure of the whole molecule. Starting off with KM-3 without any substituents on the benzoic acid as a reference, we systematically introduce cyano groups on the benzoic acid in addition to an acetylene bond at the para-position to - $\mathrm{COOH}$ and investigate the variations of photoelectrochemical properties, electronic transitions and device performances.

Studying the charge carrier dynamics, i.e. the charge/hole injection, charge recombination and dye regeneration, between the different dyes and $\mathrm{TiO}_{2}$ by various spectroscopic methods reveals that the photovoltaic performances strongly depend on a sensitive interplay between the dyes' building blocks. Quantum chemical calculations provide deeper understanding of the underlying charge transfer processes by scrutinizing the electronic and geometrical properties of the dyes.

Time-resolved femtosecond and nanosecond transient absorption measurements, for instance, allow the determination of the dynamics of charge separation and recombination. Thereby, one can focus on the generation of the TPA radical cation with its clear spectral signature beyond $580 \mathrm{~nm}$ (Fig. 2a). 
A comparison of the charge separation/recombination rates obtained by mono-exponential fitting of the time-absorption profiles of characteristic features (Fig. 2b) clearly implies a dependence on the anchoring modes. KM-6, KM-1 and KM-2 show significantly increased charge separation/recombination rates as compared with KM-3 and KM-5. An improved binding modality of the cyanobenzoic acid groups with regard to the benzoic acid groups indicates significant changes of the electronic couplings between the sensitizers and $\mathrm{TiO}_{2}$. This, on the other hand, leads to a variation of charge injection rates and recombination dynamics, which, in turn, affects the device performances.

a)

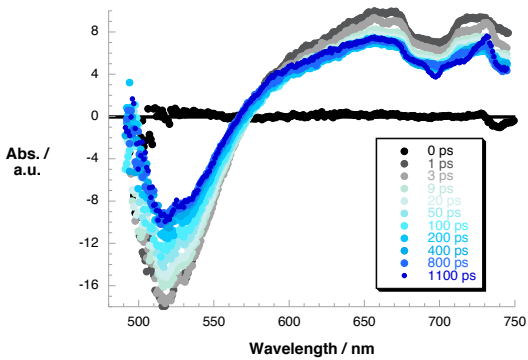

b)

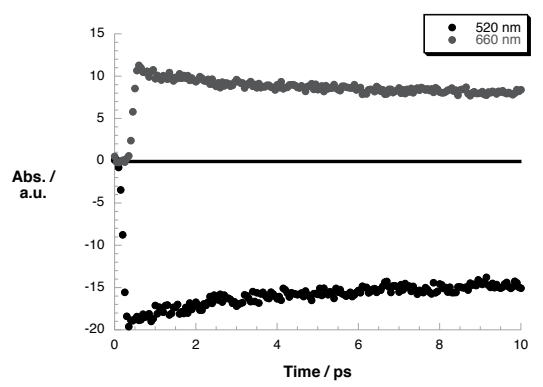

Fig. 1. a) Differential absorption spectrum obtained upon femtosecond flash photolysis $\left(\lambda_{\text {exc }}=480 \mathrm{~nm}\right)$ of a film of KM-2 on $3 \mu \mathrm{m}$ thick $\mathrm{TiO}_{2}$ with the ground-state transient bleaching between 480 and 560 $\mathrm{nm}$ and the $\mathrm{TA}^{\circ+}$ cation beyond $580 \mathrm{~nm}$. b) Time-absorption profiles illustrating the charge separation.

Furthermore, taking into account that the dyes exhibit a donor- $\pi$-spacer-acceptor architecture, we assume the charge transfer rates being dependent on the distance between donor and the acceptor and the electrons being transferred through the $\pi$-spacer. Such a charge transfer leads to a molecular-wire behavior of the spacers linking TPA to the anchoring groups. Hence, plotting the charge separation rates as a function of the distance between TPA and the anchoring groups allows for the determination of the attenuation factor $\beta$. The slopes of the linear fits of the charge separation and charge recombination rates afforded very low $\beta$ values of $0.04 \AA^{-1}$ and $0.07 \AA^{-1}$, respectively. Accordingly, the dependence of the charge separation rate on the length of the molecules turned out to be extremely low, which indicates an efficient conjugation between the donor and acceptor moieties of all dyes. Therefore, the anchoring groups are supposed to impose the most significant influence on the charge transfer rates.

\section{References}

1. M. Grätzel, 42, 1788 (2009).

2. (a) C. Chen, M. Wang, J. Li, N. Pootrakulchote, L. Alibabaei, C. Ngoc-le, J. Decoppet, J. Tsai, C. Grätzel, C. Wu, S. Zakeeruddin, M. Grätzel, ACS Nano, 3, 3103, (2009). (b) F. Gao, Y. Wang, D. Shi, J. Zhang, M. Wang, X. Jing, R. Humphry-Baker, P. Wang, S. Zakeeruddin, M. Grätzel, J. Am. Chem. Soc. 130, 10720 (2008).

3. (a) G. Zhang, H. Bala, Y. Cheng, D. Shi, X. Lv, Q. Yu, P. Wang, Chem. Commun. 2198, (2009). (b) W. Zeng, Y. Cao, Y. Bai, Y. Wang, Y. Shi, M. Zhang, F. Wang, C. Pan, P. Wang, Chem. Mater. 22, 1915 (2010).

4. A. Mishra, M. Fischer, P. Bäuerle, Angew. Chem. Int. Ed., 48, 2474 (2009) and references cited therein.

5. S. Haid, M. Marszalek, A. Mishra, M. Wielopolski, J. Teuscher, J-E. Moser, R. HumphryBaker, S. M. Zakeeruddin, M. Grätzel, P. Bäuerle, Adv. Funct. Mater. 22, 1291 (2012).

6. M. Katono, T. Bessho, M. Wielopolski, M. Marszalek, J.-E. Moser, R. Humphry-Baker, S. M. Zakeeruddin, M. Grätzel, J. Phys. Chem. C, Articles ASAP (2012). 